\title{
Prevalência de deficiência visual de perto e fatores associados: um estudo de base populacional
}

\author{
Prevalence of near vision deficiency and related \\ factors: a population-based study in Brazil
}

Wladimir Ribeiro Duarte 1

Aluísio J. D. Barros 1

Juvenal Soares Dias-da-Costa 1

Jacobo Melamed Cattan 2

\footnotetext{
1 Departamento de Medicina Social, Faculdade de Medicina, Universidade Federal de Pelotas. Av. Duque de Caxias 250, Pelotas, RS 96010-900, Brasil. fjlb@terra.com.br abarros@epidemio-ufpel.org.br jsdc@ufpel.tche.br 2 Departamento de Oftalmologia e Otorrinolaringologia, Faculdade de Medicina, Universidade Federal do Rio Grande do Sul. Rua Ramiro Barcelos 2350, Porto Alegre, RS 90035-003, Brasil. jmelamed@ncpa.ufrgs.br
}

\begin{abstract}
The objective of this article was to estimate the prevalence of presbyopia in adults $\geq 30$ years and explore associated characteristics such as demographic, socioeconomic, and individual variables. A cross-sectional, population-based study was performed with 3,007 adults $\geq 30$ years in Pelotas, Rio Grande do Sul, Brazil. Near vision acuity was evaluated using the Jaeger table, and other variables were investigated using standardized, pre-coded questionnaires. Prevalence of presbyopia was 54.7\%. When visual acuity was measured using visual corrective means, prevalence was $29.34 \%$. Age and female gender were associated with the outcome variable after controlling for confounding. Some $42.4 \%$ of the sample reported not requiring near vision for routine daily tasks. The conclusion was that presbyopia displays a high prevalence in the adult population and is directly related to increasing age, especially 45 years of age and appearing earlier in women. In some $30 \%$ of individuals who already use visual corrective means, the latter are ineffective for near vision.
\end{abstract}

Key words Presbyopia; Prevalence; Vision; Cross-Sectional Studies

Resumo Este artigo teve como objetivo estimar a prevalência de presbiopia em adultos com trinta anos ou mais e explorar características associadas, como variáveis demográficas, sócioeconômicas e individuais. Realizou-se um estudo transversal de base populacional com 3.007 adultos acima de trinta anos em Pelotas, Rio Grande do Sul, Brasil. Avaliou-se a acuidade visual para perto com a tabela de Jaeger e as outras variáveis foram investigadas através de questionários padronizados e pré-codificados. Encontrou-se uma prevalência de 54,7\% de presbiopia na amostra estudada. Quando a acuidade visual foi medida utilizando meios de correção visual, a prevalência encontrada foi de 29,3\%. As variáveis idade e sexo feminino se mostraram associadas com o desfecho, controlando-se para fatores de confusão. Cerca de 42,4\% da amostra referiram não necessitar da visão de perto nas suas tarefas habituais. Concluiu-se que a presbiopia tem elevada prevalência na população adulta e se relaciona diretamente com o aumento da idade (especialmente após os 45 anos) manifestando-se mais precocemente nas mulheres. Em $30 \%$ dos usuários de correção visual a mesma não é adequada para visão de perto.

Palavras-chave Presbiopia; Prevalência; Visão; Estudos Transversais 


\section{Introdução}

A visão se constitui em um sentido de especial relevância para a vida de relação, sendo responsável por importante parcela da informação assimilada, contribuindo decisivamente para o desenvolvimento cognitivo (Guyton \& Hall, 1996).

Para fins clínicos, divide-se a função visual em visão para longe e visão para perto. Entende-se como visão de perto aquela que compreende a definição de imagens até $1 \mathrm{~m}$ de distância dos olhos. Muitas são as causas que cursam com deficiência visual de perto - DVP (Comitê de Funções Visuais, 1988). A causa mais prevalente para DVP na população a partir da quinta década de vida é a presbiopia, seguida pelos erros refrativos, catarata e retinopatias (Abrahanson Jr., 1984; Albert \& Jakobiec, 1994).

Define-se presbiopia como um tipo de vício de refração de caráter fisiológico que tem início por volta da quarta década de vida, em que há perda da capacidade de focalizar a visão adequadamente para curtas distâncias (Esteves et al., 1993; Vaughan \& Asbury, 1983), sendo sintomática quando as reservas acomodativas são inferiores a valores entre 4,0 e 3,5 dioptrias (Adler-Gringberg, 1987; Cameron, 1976; Gil Del Rio, 1981). A presbiopia representa a causa mais freqüente de distúrbios da visão após os 40 anos de idade (Abrahamson Jr., 1984; Esteves et al., 1993; Vaughan \& Asbury, 1983).

A fisiopatogenia básica repousa sobre o senescimento dos meios acomodativos oculares, promovendo processos degenerativos no nível de músculo ciliar, zônula e cápsula do cristalino que implicam a diminuição da elasticidade e a debilitação progressiva destas estruturas (Esteves et al., 1993; Vaughan \& Asbury, 1983). Ocorrem também alterações nas propriedades bioquímicas no núcleo do cristalino provocando esclerose e aumento de sua convexidade sagital (Adler-Gringberg, 1987; Cook et al., 1994; Gil del Rio, 1981).

Estudos epidemiológicos sobre presbiopia investigam sua associação com diferentes fatores, onde a maior idade aparece como característica mais relevante (Blystone, 1999; Carter, 1982; Pointer, 1995a). Verificam-se também evidências consistentes indicando que o aparecimento mais precoce deste agravo ocorre no sexo feminino (González et al., 1992; Pointer, 1995a). Alguns estudos sugerem uma associação com a paridade, raça (Hunter Jr. \& Shipp, 1997; Ong, 1981) e condição sócio-econômica (Carter, 1982; Hunter Jr. \& Shipp, 1997), mas não há consenso. Compondo este modelo multicausal, supõe-se que fatores ambientais tais como maior exposição à luz ultravioleta e deficiência de aminoácidos, possam também estar associados com este desfecho (Jain et al., 1982).

Em relação às prevalências de presbiopia, dois estudos em adultos no continente africano encontraram prevalências distintas: Kamali et al. (1999), em um inquérito de base populacional com 2.886 adultos do meio rural de Uganda, identificou uma prevalência de $48 \%$ para esta ametropia, porém Morny (1995), a partir de registros de base ambulatorial, identificou uma prevalência de $65 \%$ em adultos de Gana.

No Brasil, em um estudo de base ambulatorial, estimou-se uma prevalência de presbiopia de $75 \%$ em indivíduos com idade superior a 40 anos, contudo, na revisão bibliográfica nacional, não se encontraram estudos de base populacional com medidas da doença (Monte, 1981).

A prevalência da presbiopia possivelmente vem aumentando em vários países do mundo $\mathrm{e}$ projeta-se um acréscimo nesta medida de ocorrência, devido, principalmente, ao fenômeno do envelhecimento populacional (Pointer, 1995b).

A presbiopia se constitui em um importante problema de saúde pública, devido à sua elevada freqüência, à existência de meio corretivo disponível e às limitações funcionais e implicações mórbidas que interferem com a qualidade de vida dos indivíduos, principalmente quando os constantes avanços tecnológicos demandam progressivamente de boa higidez visual para perto (Pointer, 1995b).

Objetivou-se, aqui, descrever a prevalência de presbiopia em adultos de 30 anos ou mais, assim como analisar e compreender as relações dos seus determinantes sociodemográficos e possíveis fatores associados à condição visual, utilizando-se uma avaliação de DVP como uma proxy para presbiopia em face da dificuldade de se realizar testes de refração no campo.

\section{Metodologia}

Realizou-se um estudo transversal de base populacional na cidade de Pelotas, Rio Grande do Sul, no período de outubro de 1999 a janeiro de 2000, envolvendo adultos com idade igual ou superior a 30 anos, residentes na zona urbana desta cidade. Excluíram-se indivíduos portadores de doença mental ou deficiência física que comprometessem o correto preenchimento dos questionários da pesquisa e a medida de acuidade visual.

O tamanho mínimo de amostra calculado para este estudo foi de 1.963 adultos, para estudar uma associação com um fator de risco 
com uma razão de quatro expostos para um não exposto, com um poder de $80 \%$, uma razão de prevalências de 2,0 e uma prevalência da morbidade de $10 \%$ nos não expostos e efeito de delineamento de 1,5, assim como estimar prevalência por faixa etária considerando como valor aceitável uma margem de erro de $1 \%$ e nível de confiança de 95\%, incluindo um acréscimo de $25 \%$ para controle de fatores de confusão, perdas e recusas (IBGE, 1997; Kamali et al., 1999; Morny, 1995). Como este estudo estava inserido em outro estudo maior, a amostra obtida excedeu o mínimo calculado.

Sortearam-se, de forma sistemática, $48 \mathrm{se}$ tores censitários, assim como a esquina de cada setor onde se iniciou a investigação, e a partir desta, os 44 domicílios a serem visitados. Estabeleceu-se um pulo de duas residências para cada uma visitada. Entrevistaram-se todos os indivíduos com idade igual ou superior a 30 anos, utilizando questionários que continham perguntas sobre variáveis sócio-econômicas, demográficas, especificidades sobre a condição visual e teste para a acuidade visual de perto com a tabela de Jaeger (Comitê de Funções Visuais, 1988).

Para mensurar a acuidade visual de perto e garantir a padronização nas aferições, adaptou-se a tabela, utilizando-se uma fita inextensível de $37 \mathrm{~cm}$ de comprimento, com uma de suas extremidades fixada à sua parte superior e a outra extremidade conectada a uma alça elástica que envolvia a cabeça do entrevistado no nível superciliar. Desta forma, com a fita em ângulo reto com a região frontal e em local com boa luminosidade, realizaram-se as mensurações binoculares simultâneas. As pessoas que utilizavam alguma forma de correção visual foram testadas com a visão desarmada e após com o meio próprio.

Definiu-se DVP como a incapacidade de visualizar todos os 14 optotipos no nível de J1 da tabela de Jaeger. Classificaram-se com visão normal os indivíduos que identificaram corretamente pelo menos um dos optotipos do nível de J1 (Comitê de Funções Visuais, 1988).

Foram estudadas as seguintes variáveis: sexo, cor da pele (branca/não branca), cor dos olhos (castanhos, verdes e azuis), idade em anos completos, paridade (número total de paras), escolaridade (número total de anos de estudo segundo as categorias utilizadas pela Fundação Instituto Brasileiro de Geografia e Estatística - IBGE), renda familiar (renda per capita em salários mínimos segundo as categorias utilizadas pelo IBGE).

Investigaram-se questões específicas sobre a condição visual, com as seguintes variáveis: autopercepção da condição visual (normal, déficit para perto, déficit para longe, déficit para perto e longe), tempo de déficit visual (de 1 a 10 anos e de 11 ou mais), uso de correção visual (sim/não), tipo de correção (óculos, lente de contato, óculos e lente de contato, outro), recomendação de correção visual (médico, balconista de óptica, familiar, outro), onde adquiriu a correção visual (óptica, vendedor ambulante, emprestado, outro), a necessidade de ver perto $(\mathrm{O}$ (a) Sr.(a) tem algo que faça seguido onde precise enxergar bem para perto? - sim/não), onde procuraria recurso caso houvesse algum problema visual (consultório privado, convênio, posto de saúde, pronto socorro, outro) e possuir plano de saúde (sim/não).

O trabalho de campo foi realizado com a participação de 24 entrevistadoras previamente submetidas a processo de seleção e posterior treinamento para a aplicação dos questionários e padronização das medidas de acuidade visual. Para o controle de qualidade, repetiram-se 50 questionários por setor. Nesta ocasião, verificaram-se algumas informações. Utilizou-se, para a avaliação da taxa de concordância, o indicador kappa. Realizou-se revisão individual da consistência de cada questionário pelos supervisores dos conglomerados.

Os dados foram digitados no programa Epi Info 6.0 duas vezes, para verificação de erros de digitação. No processo de limpeza de dados, verificou-se a consistência e amplitude das variáveis.

As análises univariadas e bivariadas foram processadas através dos programas Epi Info 6.0 e SPSS 8.0, utilizando-se como medida de efeito a razão de prevalência e seus respectivos intervalos de confiança.

A análise multivariável foi realizada através de regressão logística, de acordo com um modelo teórico (Victora et al., 1997), através do programa STATA 6.0.

Para a análise por regressão logística, utilizou-se a razão de odds como medida de efeito, cientes de que esta medida superestima a razão de prevalência quando o efeito é de risco e subestima quando o efeito é protetor. Na apresentação dos resultados, optou-se por explorar os dados referentes às medidas de razão somente para as variáveis que se mantiveram associadas ao desfecho na análise multivariável.

Os preceitos éticos que norteiam as pesquisas envolvendo seres humanos foram obedecidas na execução deste estudo, sendo este aprovado pelo comitê de ética da Faculdade de Medicina da Universidade Federal de Pelotas. 


\section{Resultados}

Obteve-se uma amostra de 3.007 pessoas - já excluídas as perdas e recusas, que totalizaram $6,8 \%$. A taxa de concordância obtida através do indicador kappa foi de 0,76 .

Os grupos etários mais prevalentes foram aqueles entre 30 e 49 anos e quando somados representaram $56,9 \%$ do total. O sexo feminino prevaleceu com $58,5 \%$. Houve predomínio de brancos com $80,7 \%$ e, quanto à cor da íris, $78,1 \%$ dos indivíduos apresentaram olhos escuros (íris de negra a castanha clara) e o restante olhos claros (íris azul ou verde). Quanto à condição sócio-econômica, 74,8\% das pessoas estavam na classe domiciliar de renda per capita igual ou inferior a 3,0 salários mínimos e $48,4 \%$ possuíam quatro anos ou menos de escolaridade. A maioria das mulheres tiveram $(45,1 \%)$ duas ou três gestações prévias (Tabela 1).

A prevalência de DVP (presbiopia) em adultos com 30 anos ou mais encontrada neste estudo foi de $54,7 \%$.

A prevalência total, por sexo e por faixa etária são apresentadas na Tabela 2 e Figura 1. Verificou-se que a freqüência de presbiopia nas mulheres foi superior à dos homens para todas as faixas de idade.

Para a variável idade, observou-se uma tendência estatisticamente significativa no aumento da prevalência de presbiopia de acordo com o aumento da mesma. Deve-se ressaltar que o maior aumento relativo de prevalência ocorreu entre as idades de 35 a 39 e 40 a 45 anos, passando de $11 \%$ para $28,4 \%$ que representa um aumento de $158 \%$. Observou-se outra diferença digna de destaque nas faixas etárias entre 40 a 44 e 45 a 50 anos com prevalência de $28,4 \%$ para $66,4 \%$, respectivamente, significando um aumento de $134 \%$.

A análise multivariável por regressão logística demonstrou a permanência no modelo das variáveis: idade e sexo como preditores associados à presbiopia. O efeito das demais variáveis (renda per capita, escolaridade, cor dos olhos, cor da pele e gestações) desapareceu quando foram controladas para a idade.

Quanto às variáveis relacionadas à condição visual, $59,1 \%$ das pessoas referiram dificuldade para visualizar a pequenas distâncias. Deste grupo, $70 \%$ referiram que esta dificuldade foi percebida entre 1 a 10 anos atrás (Tabela 3 ).

Em relação ao uso de correção visual, 54,9\% relataram o uso de algum auxílio óptico, sendo 98,1\% deste montante como usuários de óculos. Considerando o acesso ao meio corretivo, 92,5\% relataram recomendação por indicação médica, e em $94,4 \%$ das vezes o meio foi adquirido em óptica (Tabela 3 ).

Verificou-se que em $29,3 \%$ das pessoas que usavam correção visual, o meio corretivo estava inadequado para a visão de perto.

Consideraram-se présbitas funcionais os indivíduos que apresentavam dificuldade visual de perto com ou sem a utilização de meios corretivos.

Verificou-se a prevalência de presbiopia funcional em relação aos grupos etários. Os dados revelaram uma tendência do aumento com idade, ou seja, $6,1 \%$ das pessoas na faixa etária de 30 a 34 anos apresentavam dificuldade visual de perto, ao passo que $59,9 \%$ dos indivíduos no grupo dos 70 ou mais tinham a mesma condição (Tabela 4).

Realizou-se uma análise apenas entre os indivíduos que apresentavam presbiopia. Naqueles que não utilizavam meios corretivos encontrou-se uma tendência inversa, ou seja, o percentual de DVP (présbitas) diminuiu com o decorrer da idade. Nos indivíduos que utilizavam meios corretivos, os maiores percentuais de DVP (présbitas) ocorreram no grupo de 35 a 44 anos de idade e no grupo com sessenta anos ou mais (Tabela 4).

Verificou-se que $42,4 \%$ das pessoas não manifestaram necessidade de ter boa acuidade visual para perto. Ao se analisar este grupo, constatou-se que $43,2 \%$ eram présbitas, $28,6 \%$ usavam algum tipo de correção visual, sendo que, em $31,3 \%$ destas pessoas, esta correção não era efetiva de perto. Destes indivíduos, $76,6 \%$ eram brancos e $52,2 \%$ eram mulheres.

Os percentuais de homens e mulheres que referiram não necessitar de boa acuidade visual de perto na execução de tarefas rotineiras foram de $48,8 \%$ e $37,8 \%$, respectivamente. Observou-se que houve uma tendência estatisticamente significativa de que quanto maior fossem: a renda per capita, escolaridade e idade, maior era autopercepção da necessidade da utilização da visão de perto na execução das tarefas habituais. Estas variáveis permaneceram associadas ao desfecho mesmo após serem controladas para fatores de confusão.

Em relação ao acesso aos serviços de saúde, obtiveram-se os seguintes resultados: caso houvesse algum problema de etiologia ocular, $38,5 \%$ procurariam o seu convênio, $25,9 \%$, o posto de saúde, $17,2 \%$, o consultório particular, bem como $12,2 \%$ procurariam outro tipo de serviço e 5,7\% o pronto socorro. Quanto a possuir algum tipo de convênio de saúde, $42,7 \%$ dos entrevistados relatam estar conveniados. 


\section{Discussão}

Nesta investigação, priorizou-se um estrito rigor metodológico que resultou em dados de alta confiabilidade, confirmados pelas reduzidas perdas e recusas, ausência de vieses de seleção, mensurações adequadas aos fins propostos e utilização de métodos de aleatorização e amostragem sustentados estatisticamente, além do controle intensivo de qualidade em todas as fases da pesquisa (Barros \& Victora, 1994; Pereira, 1995).

Acredita-se que neste estudo a medida da DVP foi a medida do fenômeno presbiópico, porém a definição de presbiopia deverá ser vista sob a crítica de duas importantes limitações. Primeiro, o método utilizado para o diagnóstico de presbiopia (tabela de Jaeger) está sujeito a erros de medida, apesar do extremo cuidado que tivemos em sua utilização no campo. Segundo, podem ter sido classificadas como presbiopia algumas condições específicas que comprometam a visão de perto, como ametropias de alto grau, catarata avançada, retinopatias graves e baixa visão (Comitê de Funções Visuais, 1988).

Por outro lado, acredita-se que os erros de classificação foram minimizados pela medida visual binocular simultânea e porque as patologias que poderiam ser erroneamente classificadas como presbiopia necessitariam estar em grau avançado de acometimento.

Outras limitações neste estudo foram quanto à impossibilidade de avaliar fatores que podem estar envolvidos na determinação de presbiopia (clima, fatores nutricionais, exposição à luz solar).

Ressalta-se que o número de 3.007 entrevistados foi superior à amostra mínima necessária para o teste estatístico de associação, conferindo ao mesmo um poder de $99 \%$, já acrescido os $25 \%$ de perdas, recusas e fatores de confusão, considerando um efeito de delineamento de 1,5 .

Encontrou-se uma prevalência de 54,7\% de presbiopia em adultos com 30 anos ou mais, resultado compatível com os encontrados na literatura, em que a prevalência geral se situa entre $48 \%$ e $65 \%$ (Kamali et al., 1999; Monte, 1981; Morny, 1995). Porém, ressalva-se que a faixa etária e a origem das populações (ambulatorial) destes estudos pode explicar as diferenças encontradas. Este estudo tem o mérito de fazer uma medida populacional e por faixas de idade.

A idade, classicamente apontada como fator preponderante para o aparecimento de presbiopia, mostrou-se como o preditor mais forte-
Tabela 1

Distribuição de freqüências de variáveis sociodemográficas e prevalências brutas de deficiência visual de perto (DVP). Pelotas, Rio Grande do Sul, Brasil, 2000.

\begin{tabular}{|c|c|c|c|}
\hline Variável & Freqüência & $\%$ & $\begin{array}{l}\text { Prevalência } \\
\text { de DVP (\%) }\end{array}$ \\
\hline Idade $(n=3.007)$ & & & $p<0,001$ \\
\hline 30 a 34 anos & 413 & 13,7 & 10,4 \\
\hline 35 a 39 anos & 434 & 14,4 & 11,0 \\
\hline 40 a 44 anos & 423 & 14,1 & 28,4 \\
\hline 45 a 49 anos & 443 & 14,8 & 66,4 \\
\hline 50 a 54 anos & 347 & 11,5 & 80,5 \\
\hline 55 a 59 anos & 251 & 8,3 & 87,6 \\
\hline 60 a 64 anos & 244 & 8,1 & 93,4 \\
\hline 65 a 69 anos & 171 & 5,7 & 92,5 \\
\hline 70 ou mais & 281 & 9,3 & 93,1 \\
\hline Sexo $(n=3.011)$ & & & $p<0,001$ \\
\hline feminino & 1.760 & 58,5 & 59,3 \\
\hline masculino & 1.251 & 41,5 & 48,4 \\
\hline \multicolumn{4}{|l|}{ Cor da pele $(n=3.011)$} \\
\hline branca & 2.431 & 80,7 & 55,7 \\
\hline não branca & 580 & 19,3 & 51,1 \\
\hline \multicolumn{4}{|l|}{ Cor dos olhos $(n=1.285)^{\star}$} \\
\hline castanhos & 1.004 & 78,1 & 88,5 \\
\hline verdes & 147 & 11,4 & 87,0 \\
\hline azuis & 134 & 10,4 & 92,0 \\
\hline \multicolumn{4}{|l|}{ Renda per capita $(n=2.953$ ) } \\
\hline 0 a 1,0 salário mínimo & 1.075 & 36,4 & 50,2 \\
\hline 1,1 a 3,0 salários mínimos & 1.134 & 38,4 & 57,0 \\
\hline 3,1 a 6,0 salários mínimos & 415 & 14,0 & 56,0 \\
\hline 6,1 a 10,0 salários mínimos & 173 & 5,9 & 60,4 \\
\hline 10,1 ou mais salários mínimos & 156 & 5,3 & 58,6 \\
\hline Escolaridade $(n=2.982)$ & & & $p<0,001 * \star$ \\
\hline 0 a 4a série do 1o grau & 1.446 & 48,4 & 65,9 \\
\hline 5ạ a 7ạ série do 1ㅇ grau & 567 & 19,0 & 44,4 \\
\hline 8o ano do 1ㅇ grau ao 2o ano do 2o grau & 506 & 16,9 & 44,8 \\
\hline 3o ano do 2o grau ao 3o ano do 3o grau & 327 & 10,9 & 39,0 \\
\hline 4o ano do 3 o grau ou mais & 143 & 4,8 & 52,0 \\
\hline Número de gestações ( $n=1.495)$ & & & $p<0,001 * \star$ \\
\hline 0 gestações & 167 & 10,6 & 48,7 \\
\hline 1 gestação & 235 & 14,9 & 40,6 \\
\hline 2 gestações & 393 & 24,9 & 56,2 \\
\hline 3 gestações & 318 & 20,2 & 54,3 \\
\hline 4 gestações & 175 & 11,1 & 51,2 \\
\hline 5 ou mais gestações & 289 & 18,3 & 77,0 \\
\hline
\end{tabular}

* Esta variável foi coletada somente para pessoas com 50 anos ou mais. ** A associação destas variáveis com DVP deve ser avaliada com cuidado, em função do forte confundimento por idade. Após ajuste para a idade, estas variáveis deixaram de estar significativamente associadas com o desfecho. 
Tabela 2

Prevalência de deficiência visual de perto (DVP) de acordo com a faixa etária a cada cinco anos: geral e por sexo. Pelotas, Rio Grande do Sul, Brasil, 2000.

\begin{tabular}{|c|c|c|c|c|c|c|}
\hline \multirow[t]{2}{*}{ Idade (anos) } & \multicolumn{2}{|c|}{ Feminino } & \multicolumn{2}{|c|}{ Masculino } & \multicolumn{2}{|c|}{ Total } \\
\hline & Prevalência (\%) & IC $95 \%$ & Prevalência (\%) & IC $95 \%$ & Prevalência (\%) & IC 95\% \\
\hline $30-34$ & 11,3 & $7-15$ & 9,3 & $5-13$ & 10,4 & $7-13$ \\
\hline $35-39$ & 13,5 & $10-18$ & 7,8 & $3-11$ & 11,0 & $7-14$ \\
\hline $40-44$ & 31,9 & $26-38$ & 23,3 & $16-30$ & 28,4 & $24-32$ \\
\hline $45-49$ & 71,5 & $65-77$ & 58,7 & $51-65$ & 66,4 & $62-71$ \\
\hline $50-54$ & 86,5 & $81-91$ & 72,1 & $64-80$ & 80,5 & $76-84$ \\
\hline $55-59$ & 90,7 & $85-95$ & 83,3 & $75-90$ & 87,6 & $83-91$ \\
\hline $60-64$ & 94,4 & $90-98$ & 91,8 & $85-97$ & 93,4 & $90-96$ \\
\hline $65-69$ & 93,7 & $88-98$ & 90,8 & $83-98$ & 92,5 & $88-96$ \\
\hline 70 ou + & 94,3 & $90-97$ & 90,9 & $84-97$ & 93,1 & $90-96$ \\
\hline Total & 59,3 & $57-61$ & 48,5 & $45-51$ & 54,7 & $53-56$ \\
\hline
\end{tabular}

Figura 1

Prevalência de deficiência visual de perto (presbiopia) de acordo com idade e sexo.

Pelotas, Rio Grande do Sul, Brasil, 2000.

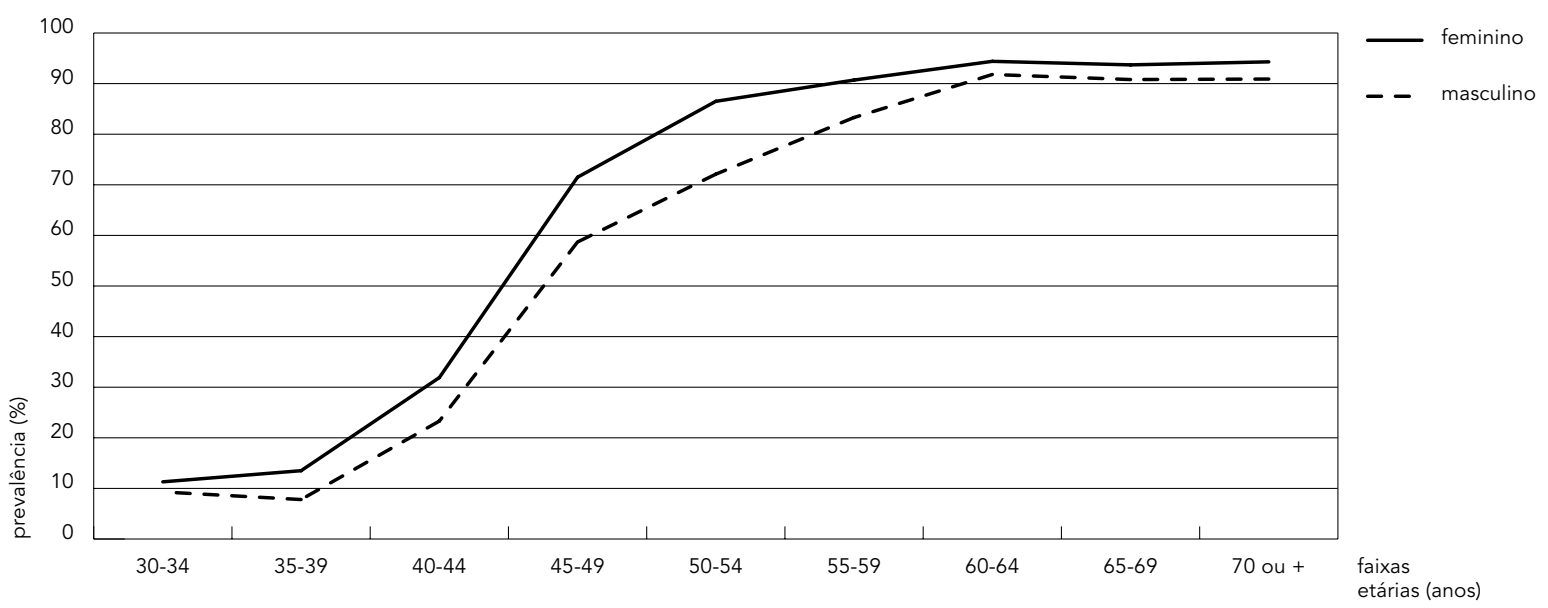


mente associado ao desfecho, apresentando uma razão de prevalências de nove vezes para a idade de 70 anos ou mais, comparada com a faixa etária de 30 a 34 anos (Blystone, 1999; González et al., 1992).

Corrobora para a tese de que a ocorrência da presbiopia seja um fenômeno essencialmente cronológico o fato de que se encontrou uma tendência linear entre presbiopia e idade.

A noção de que o aparecimento de presbiopia é mais precoce no sexo feminino foi suportada pelos dados desta investigação, pois se verificou que as mulheres tiveram um risco global de $22 \%$ a mais de serem acometidas que os homens (Morny, 1995). Verificou-se que esta diferença entre os sexos se fez observar mais claramente na transição da quarta para a quinta década de vida. Contudo, esta diferença diminui sensivelmente a partir da sexta década, em que a prevalência deste agravo é praticamente a mesma para ambos os sexos (Pointer, 1995a).

Outro aspecto relevante desta investigação foi o elevado percentual de pessoas $(43,2 \%)$ que afirmaram não necessitar da visão para perto na execução de suas atividades diárias. Esta população se mostrou predominantemente mais pobre, menos escolarizada, pertencente ao sexo masculino e mais jovem.

Este perfil indica que grupos menos favorecidos têm menor percepção da relevância da higidez visual como pré-requisito para o trabalho e implementação cognitiva. Uma possível explicação para este achado pode residir no fato de que estes grupos não exerçam atividades mais refinadas que demandem boa visão de perto.

\begin{tabular}{|c|c|c|c|}
\hline $\begin{array}{l}\text { Distribuição de freqüências de } \\
\text { Pelotas, Rio Grande do Sul, Bra }\end{array}$ & ao sistema vi & & \\
\hline Variável & Freqüência & $\%$ & $\begin{array}{l}\text { Prevalência } \\
\text { de DVP* (\%) }\end{array}$ \\
\hline \multicolumn{4}{|c|}{ Percepção da higidez visual $(n=3.004)$} \\
\hline Normal & 914 & 30,4 & 12,6 \\
\hline Dificuldade para perto & 925 & 30,8 & 73,0 \\
\hline Dificuldade para longe & 316 & 10,5 & 38,7 \\
\hline Dificuldade para ambos & 849 & 28,3 & 86,1 \\
\hline \multicolumn{4}{|c|}{ Tempo de dificuldade visual $(n=1.718)$} \\
\hline 1 a 10 anos & 1.194 & 69,5 & 74,6 \\
\hline 11 a 87 anos & 524 & 30,5 & 89,5 \\
\hline \multicolumn{4}{|c|}{ Uso de correção visual $(n=3.009)$} \\
\hline Sim & 1.653 & 54,9 & 78,6 \\
\hline Não & 1.356 & 45,1 & 26,0 \\
\hline \multicolumn{4}{|c|}{ Tipo de correção visual $(n=1.652)$} \\
\hline Óculos & 1.621 & 98,1 & 78,7 \\
\hline Lente de contato & 16 & 1,0 & 62,5 \\
\hline Óculos e lente de contato & 10 & 0,6 & 77,8 \\
\hline Outro tipo de correção & 05 & 0,3 & 100 \\
\hline \multicolumn{4}{|c|}{ Recomendação de correção visual $(n=1.649)$} \\
\hline Médico & 1.526 & 92,5 & 77,6 \\
\hline Balconista de ótica & 35 & 2,1 & 88,6 \\
\hline Familiar & 26 & 1,6 & 88,0 \\
\hline Outro & 62 & 3,8 & 93,2 \\
\hline \multicolumn{4}{|c|}{ Aquisição de correção visual $(n=1.648)$} \\
\hline Ótica & 1.556 & 94,4 & 77,8 \\
\hline Vendedor ambulante & 25 & 1,5 & 95,8 \\
\hline Emprestado & 24 & 1,5 & 91,3 \\
\hline Outro & 43 & 2,6 & 90,5 \\
\hline
\end{tabular}

* DVP = Deficiência visual de perto.

Tabela 4

Freqüência de deficiência visual de perto (DVP) incluindo usuários de correção visual, segundo a faixa etária. Pelotas, Rio Grande do Sul, Brasil, 2000.

\begin{tabular}{|c|c|c|c|c|c|c|}
\hline \multirow[t]{2}{*}{ Idade (anos) } & \multicolumn{2}{|c|}{ Présbitas funcionais } & \multicolumn{2}{|c|}{$\begin{array}{l}\text { Présbitas que não } \\
\text { usam correção }\end{array}$} & \multicolumn{2}{|c|}{$\begin{array}{l}\text { Présbitas mesmo } \\
\text { com correção }\end{array}$} \\
\hline & Total & DVP (\%) & Total & DVP (\%) & Total & DVP (\%) \\
\hline $30-34$ & 394 & 6,1 & 41 & 43,9 & 22 & 22,7 \\
\hline $35-39$ & 410 & 7,6 & 45 & 48,9 & 20 & 35,0 \\
\hline $40-44$ & 407 & 17,4 & 115 & 38,3 & 69 & 36,2 \\
\hline $45-49$ & 425 & 32,7 & 281 & 27,8 & 190 & 25,8 \\
\hline $50-54$ & 329 & 33,4 & 265 & 20,4 & 206 & 24,8 \\
\hline $55-59$ & 242 & 36,8 & 212 & 17,4 & 173 & 28,9 \\
\hline $60-64$ & 229 & 41,9 & 214 & 14,0 & 176 & 32,9 \\
\hline $65-69$ & 160 & 42,5 & 148 & 15,5 & 122 & 35,2 \\
\hline 70 ou mais & 262 & 59,9 & 242 & 12,0 & 205 & 58,0 \\
\hline Total & 2.858 & 27,5 & 1.563 & 21,4 & 1.183 & 34,4 \\
\hline
\end{tabular}


Cabe ressaltar que se considera elevada a prevalência de $29,3 \%$ de pessoas com correção inadequada para a visão de perto, e nota-se que os melhores índices de correção foram observados entre as idades de 45 a 60 anos. As possíveis hipóteses para este fato seriam: a demora no diagnóstico e tratamento entre os mais jovens e as falhas no acompanhamento da evolução do indivíduo mais idoso. Neste caso, deve-se ajustar, periodicamente, a terapêutica com lentes corretivas de acordo com a evolução da presbiopia. Esta medida é eficaz e necessária, além de possuir as seguintes vantagens: execução simples, custo baixo e ter grande impacto na melhoria da visão.
Acredita-se que políticas de saúde voltadas às pessoas mais idosas, de menor renda e escolaridade com enfoque nas ações preventivas podem ser efetivas quanto à melhoria das condições de vida destas populações, assim sugere-se que a triagem oftalmológica com a tabela de Jaeger para identificar presbiopia em pessoas com idade superior a 30 anos seria benéfica, uma vez que ações desta natureza têm impacto positivo sobre o processo saúde e doença a longo prazo, pois atualmente se dispõe de testes diagnósticos com boa acurácia e os meios corretivos são disponíveis a baixo custo.

\section{Agradecimentos}

Às agências: Coordenação de Aperfeiçoamento de Pessoal de Nível Superior (CAPES) pela bolsa de Mestrado; Fundação de Amparo à Pesquisa do Estado do Rio Grande do Sul (FAPERGS) e Pró-Reitoria de Extensão da Universidade Federal de Pelotas, pelo auxílio financeiro, indispensável na execução deste estudo; aos laboratórios Varilux e Essilor, por fornecer os instrumentos para a acuidade visual (tabelas de Jaeger); aos doutores: Sérgio Kwitko (oftalmologista), Jorge Umberto Béria (epidemiologista) e Ana Cláudia Gastal Fassa (epidemiologista), pela orientação crítica e indispensável na construção deste artigo. 


\section{Referências}

ABRAHAMSON Jr., I. A., 1984. Eye changes after forty. American Family Physician, 29:171-181.

ADLER-GRINGBERG, D., 1987. Questioning our classical understanding of accommodation and presbyopia. American Journal of the Optometry and Physiological Optics, 63:571-580.

ALBERT, D. M. \& JAKOBIEC, F. A., 1994. Principles and practice of ophthalmology: Basic sciences. In: The Epidemiology of Age-Related Cataract (S. E. Hankinson, ed.), pp. 1256-1257, Philadelphia: W. B. Saunders.

BARROS, F. C. \& VICTORA, C. G., 1994. Epidemiologia da saúde infantil. In: Preparação do Protocolo (L. F. Barros \& C. G. Victora, org.), pp. 10, São Paulo: Editora Hucitec/Fundo das Nações Unidas para a Infância.

BLYSTONE, P. A., 1999. Relationship between age and presbyopic addition using a sample of $3.645 \mathrm{ex}-$ aminations from a single private practice. Journal of the American Optometric Association, 70:505-508.

CAMERON, J. H., 1976. Headaches in relation to the eyes. Medical Journal of Australia, 1:292-294.

CARTER, M. E., 1982. Predictable visual responses to increasing age. Journal of the American Optometric Association, 53:31-36.

COMITÊ DE FUNÇÕES VISUAIS - CONCILIUM OPHTALMOLOGICUM UNIVERSALE, 1988. Medida padrão da acuidade visual. Arquivos Brasileiros de Oftalmologia, 51:203-213.

COOK, C. A.; KORETZ, J. F.; PFAHNL, A.; HYUN, J. \& KAUFMAN, P. L., 1994. Aging of the human crystalline lens and anterior segment. Vision Research, 34:2945-2954.

ESTEVES, J.; KWITKO, S. \& TELICHEVISK, N., 1993. Condutas em Oftalmologia. Porto Alegre: Editora Artes Médicas.

GIL DEL RIO, E., 1981. Óptica Fisiológica Clínica. Barcelona: Toray.

GONZALEZ, M. F.; AVILA, C. P.; MARRERO, X. M. H. \& GENER, E. V., 1992. Corrección de la presbicia en nuestro medio. Revista Cubana de Oftalmología, 5:91-99.

GUYTON, A. C. \& HALL, J. E., 1996. Taskbook of Medical Physiology. Philadelphia: W. B. Saunders.
HUNTER Jr., H. \& SHIPP, M., 1997. A study of racial differences in age at onset and progression of presbyopia. Journal of the American Optometric Association, 68:171-177.

IBGE (Fundação Instituto Brasileiro de Geografia e Estatística), 1997. Contagem da População 1997. Resultados Relativos a Sexo da População e Situação de Unidade Domiciliar 1996. Rio de Janeiro: IBGE.

JAIN, I. S.; RAM, J. \& GUPTA, A., 1982. Early onset of presbyopia. American Journal of Optometry and Physiological Optics, 595:1002-1004.

KAMALI, A.; WHITWORTH, J. A.; RUBERANTUARI, A.; MULWANYI, F.; ACAKARA, M.; DOLIA, P. \& JOHNSON, G., 1999. Causes and prevalence of non-vision impairing ocular conditions among a rural adult population in SW Uganda. Ophthalmic Epidemiology, 6:41-48.

MONTE, F. Q., 1981. Presbiopia: Conceitos atuais. Ceará Médico, 3:21-30.

MORNY, F. K., 1995. Correlation between presbyopia, age and number of births of mothers in the Kumasi area of Ghana. Ophthalmic and Physiological Optics, 15:463-466.

ONG, J., 1981. Southeastern Asian refugee's presbyopia. Perceptual and Motor Skills, 53:667-670.

PEREIRA, M. G., 1995. Epidemiologia: Teoria e prática. In: Aferição dos Eventos (M. G. Pereira, org.), pp. 368-373, Rio de Janeiro: Guanabara Koogan.

POINTER, J. S., 1995a. Broken down by age and sex. The optical correction of presbyopia revisited. Ophthalmic and Physiological Optics, 15:439-443.

POINTER, J. S., 1995b. The presbyopic add magnitude and distribution in a historical context. Ophthalmic and Physiological Optics, 15:235-240.

VAUGHAN, D. \& ASBURY, T., 1983. Oftalmologia geral. In: Ótica e Refração (O. W. White, org.), pp. 375-379, São Paulo: Editora Atheneu.

VICTORA, C. G.; HUTTLY, S. R.; FUCHS, S. C. \& OLINTO, M. T. A., 1997. The role of conceptual frameworks in epidemiological analysis: A hierarchical approach. International Journal of Epidemiology, 26:224-227.

Recebido em 26 de outubro de 2001

Versão final reapresentada em 2 de outubro de 2002

Aprovado em 11 de dezembro de 2002 